\title{
Evaluation of emotional, behavioral problems and family functioning in adolescents with chronic gastritis
}

\author{
Gonca Özyurt, M.D. Assistant Professor ${ }^{a}$, Yeliz Çă̆an-Appak, M.D. ${ }^{b}$, \\ Miray Karakoyun, M.D. ${ }^{c}$ and Maşallah Baran, M.D. Associate Professor ${ }^{d}$
}

\begin{abstract}
Objectives. The aim of the present study was to investigate psychiatric symptoms in adolescents diagnosed as having chronic gastritis, and to evaluate family functioning.

Methods. The population consisted of adolescents who were diagnosed endoscopically and histopathologically as having chronic gastritis without additional chronic disease. The anxiety levels, depression levels, and emotional and behavioral symptoms of the adolescents were measured using the Screen for Child Anxiety Related Emotional Disorders (SCARED), Beck Depression Inventory (BDI), and Strengths and Difficulties Questionnaire (SDQ). Family functioning was evaluated using the Family Assessment Device (FAD).

Results. Fifty eight adolescents were included to study. According to the SDQ subscale results, adolescents with gastritis had more problems in emotional, hyperactivity, and peer relations areas, but the results in conduct problems and prosocial behaviors were normal. Levels of all subscales of Family Assessment Device were higher than 2, showing problems in family functioning.

Conclusions. This study suggests that adolescents with chronic gastritis experience more difficulties in peer relations and family functioning, and they express more emotional problems.

Key words: adolescent, anxiety, depression, family functioning, gastritis.
\end{abstract}

http:/ / dx.doi.org/10.5546/ aap.2019.eng.e110

To cite: Özyurt G, Çağan-Appak Y, Karakoyun M, Baran M. Evaluation of emotional, behavioral problems and family functioning in adolescents with chronic gastritis. Arch Argent Pediatr 2019;117(2):e110-e114.

\section{INTRODUCTION}

In adolescence, biopsychosocial development includes changes in parental and peer relations, schools, and cognitive functioning. ${ }^{1}$ Adolescents with chronic physical illness (e.g. asthma, diabetes, epilepsy) feel apprehensive about chronic disease related limitations in their lives. ${ }^{2}$ Approximately $20 \%$ of adolescents have a chronic physical illness that affects themselves, their families, and society. ${ }^{3,4}$ Chronically ill children and adolescents often have emotional or behavioral symptoms as a result of their condition. ${ }^{5}$ Chronic disease may also profoundly affect children's families, requiring important daily adaptation for children and their families. ${ }^{6}$

Adolescents with chronic gastrointestinal (GI) diseases were examined in recent studies and emotional problems were seen more frequently in adolescents with coeliac disease ${ }^{7,8}$ Gastritis, which is inflammation of the gastric mucosa, causes abdominal pain, nausea, and dyspepsia. ${ }^{9,10}$ Goodwin et al., ${ }^{10}$ demonstrated a consistent association between gastritis and psychiatric symptoms in adults. ${ }^{10}$ In the current literature, psychiatric symptoms and family functioning in adolescents with chronic gastritis has not been investigated.

In the present study, the primary aim was to investigate the psychiatric symptoms of adolescents with chronic gastritis. The second objective was to evaluate family functioning in adolescents with gastritis.

\section{POPULATION AND METHODS Population}

The participants comprised of adolescents who presented to the pediatric gastroenterology at Izmir Tepecik Training and Research Hospital from September 2017 to December 2017 and received clinical and histopathologic diagnoses of chronic gastritis. Adolescents who presented with GI symptoms such as chronic abdominal pain, bloating, nausea, and other symptoms of gastritis, which was diagnosed via endoscopy and gastric biopsy, were 
included in the study. ${ }^{11}$ Eligible participants were aged between 12 and 18 years. Adolescents with any additional chronic diseases were excluded. Psychiatric symptoms related with psychiatric or neurologic disorders may be confusing when determining psychiatric symptoms related with gastritis; therefore, patients with psychiatric disorders were excluded.

Ethics committee approval for the study was obtained from Izmir Tepecik Research and Training Hospital Clinical Trials Ethics Committee. Verbal and written informed consents of the mothers and their children who participated in the study were received after the design and procedure of study were explained. The study was conducted in compliance with the latest version of the Declaration of Helsinki.

\section{Assessment of psychiatric symptoms and family functioning}

The Screen for Child Anxiety Related Emotional Disorders (SCARED): SCARED is a 41-item scale that aims to indicate how children felt in the last three months. "Not True or Hardly Ever True," "Somewhat True or Sometimes True," and "Very True or Often True" are the response options. ${ }^{18}$ Reports from the child (SCARED-C) and parent's (SCARED-P) can be used. Turkish version's validity and reliability study was conducted by Çakmakçı. ${ }^{19}$ The cut-off point of SCARED is suggested as 25.

Beck Depression Inventory (BDI): The BDI was developed by Beck ${ }^{16}$ and the Turkish versions were assessed by Hisli. ${ }^{17}$ The BDI has 21 items for evaluating depression symptoms in the last two weeks. Each item is scored in a scale of 0-3 (total score: 0-63). The cut-off point of the BDI is suggested as 17 for Turkish samples.

The Strengths and Difficulties Questionnaire (SDQ): SDQ was developed by Goodman in 1997. ${ }^{12}$ In this scale, (1) emotional problems, (2) peer problems, (3) behavioral problems, (4) attention deficit and hyperactivity, and (5) social behaviors are evaluated with 25 items. Each subscale score is examined within itself and the total of the first four subscales gives the "total difficulty score." The reliability and validity of the Turkish version of SDQ has previously been shown by Güvenir et al. ${ }^{13}$

The McMaster Family Assessment Device (FAD): FAD is a 60 -item family functioning screening measurement, which was developed by Epstein, Bolwin, and Bishop (1983). ${ }^{14}$ Problem solving, communication, roles, affective responsiveness, affective involvement, behavior control, and general functions are seven subscales of FAD whose scores range between 1.00 (healthy) and 4.00 (non-healthy). Scores higher than 2 are considered as non-healthy. Bulut (1990) translated the Turkish forms and its validity and reliability were confirmed by Bulut. ${ }^{15}$

\section{Statistical analysis}

The Statistical Package for the Social Sciences (SPSS) 18.0 (SPSS, Inc., Chicago, IL, USA) program was used for statistical analysis of the data obtained in the study. Certain sociodemographic and clinical categorical variables were evaluated using number and percentage values. The scores of the scales are explained with mean \pm standard deviation (SD).

\section{RESULTS}

During the study period, 214 children and adolescents presented to our clinic with symptoms of gastritis. One hundred twentyfour of these patients were aged between 12 and 18 years. Seven patients were excluded from the study because of the detection of normal histopathology, and 38 were excluded due to the presence of other chronic disease (diabetes, asthma, celiac disease, inflammatory bowel disease, reflux/esophagitis). Six of the remaining 79 patients were immigrants and did not have sufficient ability with the Turkish language to complete the scales. Fifteen patients refused to participate in the study. Thus, 58 adolescents were included in the final analysis.

The age, sex, mothers' age, educational level, parental marital status, and school success of the adolescents are shown in Table 1.

The distribution of symptoms in the adolescents with gastritis was as follows: epigastric pain $(n=14)$, abdominal pain $(n=12)$, epigastric pain and vomiting $(n=9)$, epigastric pain and bloating $(n=6)$, epigastric pain and nausea $(n=6)$, abdominal pain and vomiting $(\mathrm{n}=3)$, abdominal pain and nausea $(\mathrm{n}=3)$, nausea and vomiting $(\mathrm{n}=2)$, vomiting $(\mathrm{n}=2)$, and bloating and vomiting $(\mathrm{n}=1)$.

The scores and the reference values are shown in Table 2. Mean values for SCARED-C, SCARED-P and Beck Depression Scale were within the normal ranges.

According to the SDQ subscale results, adolescents with gastritis had more problems in emotional problems (borderline), conduct problems (borderline), hyperactivity (borderline), 
and peer relations (abnormal) areas. The mean total score of the SDQ was found as abnormal.

Family problems were compared using FAD with the reports of the mothers. The families whose children had gastritis reported more problems in all subscales (problem solving, communication, roles, affective involvement, affective responsiveness, behavior control, general functioning areas) of FAD (score $>2$ in all subscales). These results are shown in Table 2.

\section{DISCUSSION}

In the current study, there were two main findings. First, gastritis in adolescents appears to be related with significantly increased emotional problems and peers relation problems. Second, difficulties of family functioning in adolescents with gastritis were significant.

This is the first study to investigate emotional and behavioral problems in adolescents with gastritis. A recent population-based study whose sample consisted of more than 4000 adults aged between 18 and 79 years in the German National Health Interview and Examination Survey showed that psychiatric symptoms were significantly more frequent in adults with gastritis. ${ }^{10}$ Psychiatric symptoms were also shown in rats after iodo acetamide-induced gastritis. ${ }^{20,21}$ Two main possibilities exist for the mechanisms of these associations. First, a causal relationship may occur between gastritis and psychiatric symptoms. Abdominal pain, bloating symptoms of gastritis may provoke emotional problems.

TABLE 1. Sociodemographic characteristics of the patients

\begin{tabular}{lc}
\hline Characteristic & Patients $(\mathbf{n = 5 8})$ \\
\hline Age, years (mean \pm SD) & $15.51 \pm 1.60$ \\
Female & $36(62 \%)$ \\
Male & $22(38 \%)$ \\
Maternal age, years (mean \pm SD) & $38.86 \pm 6.25$ \\
Maternal education & \\
$\quad \leq 8$ years & $33(57 \%)$ \\
$>8$ years & $25(43 \%)$ \\
Marital status & \\
Married & $54(93 \%)$ \\
Divorced & $4(7 \%)$ \\
Economic status & \\
$>5000$ TRQ $(>1250$ USD) & $27(47 \%)$ \\
2000-5000 TRQ & \\
(500-1250 USD) & $19(33 \%)$ \\
$<2000$ TRQ (500 USD) & $12(20 \%)$ \\
School success & \\
Good & $27(47 \%)$ \\
Poor & $31(53 \%)$ \\
\hline
\end{tabular}

Gastrointestinal activity differences can cause changes in brain function with potential neuronal and inflammatory pathways. ${ }^{22-24}$ Konturek et al., ${ }^{24}$ demonstrated that increased anxiety might provoke gastritis via a gut-brain neuronal axis in the stomach part. ${ }^{24}$ Psychological stress may cause changes in functionality of GI system (increasing secretion, motility, inflammation). ${ }^{25}$ Secondly, common genetic or environmental risks may be present in the development of both gastritis and emotional symptoms. Independent from mechanisms, there was an association between emotional or psychiatric symptoms and gastritis in adolescents as shown in recent literature. ${ }^{26}$ A recent study investigated childhood psychiatric symptoms in functional constipation using the SDQ, and emotional and peer problem subscale scores were found higher in patients with gastritis, in line with the results in the present study. ${ }^{27}$

Adolescence is a difficult period of life for both adolescents and families. In addition to having GI symptoms, chronic gastritis may complicate this process and family life. Parents of children with conditions such as cerebral palsy, ${ }^{28}$

TABLE 2. Anxiety, depression levels, scores of strengths and difficulties questionnaire, and family assessment device subscales

\begin{tabular}{lcc}
\hline Questionnaire & Score & Reference value \\
\hline SCARED-C & $21.76 \pm 15.01$ & $0-25$ \\
SCARED-P & $21.37 \pm 13.51$ & $0-25$ \\
Beck Depression Scale & $14.18 \pm 10.78$ & $0-17$ \\
& & Normal/ \\
& & Borderline/ \\
SDQ & & Abnormal \\
\hline Emotional problems scale & $5.57 \pm 2.23$ & $0-5 / 6 / 7-10$ \\
Conduct problems scale & $3.19 \pm 1.99$ & $0-3 / 4 / 5-10$ \\
Hyperactivity scale & $5.87 \pm 1.73$ & $0-5 / 6 / 7-10$ \\
Peer problems scale & $5.41 \pm 1.37$ & $0-3 / 4-5 / 6-10$ \\
Prosocial scale & $7.75 \pm 1.83$ & $6-10 / 5 / 0-4$ \\
Total scale & $20.06 \pm 4.11$ & $0-15 / 16-19 / 20-40$ \\
FAD & & \\
\hline Problem solving & $2.38 \pm 0.51$ & $0-2$ \\
Communication & $2.25 \pm 0.42$ & $0-2$ \\
Roles & $2.44 \pm 0.53$ & $0-2$ \\
Affective involvement & $2.37 \pm 0.49$ & $0-2$ \\
Affective responsiveness & $2.47 \pm 0.40$ & $0-2$ \\
Behavior control & $2.32 \pm 0.40$ & $0-2$ \\
General functioning & $2.43 \pm 0.36$ & $0-2$ \\
\hline
\end{tabular}

SDQ: Strengths and Difficulties Questionnaire. FAD: Family Assessment Device; SCARED C: Child Screen for Anxiety Related Emotional Disorders Child version. SCARED P: Screen for Child Anxiety Related Emotional Disorders Parent version. 
asthma, abdominal pain, and headaches, ${ }^{29}$ usually have significant levels of psychological distress, and children have emotional and behavioral problems. This distress in parents and children may cause difficulties in family functioning, such as it was indicated in current study. Relations and roles within the family may become complicated as a result of this distress. Parental attitudes and affective involvement may become difficult in these families. In a recent study, over-parenting attitudes were found higher in functional constipation. ${ }^{27}$ Psychosocial interventions that target families of adolescents with gastritis may be considered as another part of treatment.

The fact that we only used information from the mothers and adolescents themselves in the present study could have affected the objectivity of the present study; receiving information from teachers could make the results more objective. The case group had serious symptoms, as such they were taken to endoscopy; these serious problems might have affected the patients' results. These important statistical differences may be related with serious gastritis symptoms. Only 58 patients were included to study from 124 patients with gastritis symptoms. The lost was over $50 \%$. This is another limitation of the study.

To the best of our knowledge, this is the first study to examine the association between gastritis and psychiatric findings in adolescents. Our findings suggest that adolescents with gastritis have emotional and behavioral problems and they have difficulties in peer relations. Family functioning was affected by gastritis as a chronic disease. Further studies are merited to investigate and help to determine a common etiology of gastritis and psychiatric symptoms by identifying potential underlying physiologic pathways of these links. Psychosocial interventions targeting psychiatric and emotional problems that may affect adolescents' ability to cope effectively with gastritis may be efficacious in reducing symptoms. Parents may also contribute to these interventions for solving family problems.

\section{REFERENCES}

1. Rech RR, Halpern R, Tedesco A, Santos DF. Prevalence and characteristics of victims and perpetrators of bullying. $J$ Pediatr (Rio J). 2013; 89(2):164-70.

2. Balázs J, Miklòsi $\mathrm{M}$, Keresztény $\mathrm{A}$, Hoven $\mathrm{CW}$, et al. Comorbidity of Physical and anxiety Symptoms in Adolescent: Functional Impairment, Self Related Health and Subjective Well-Being. Int J Environ Res Public Health. 2018 Aug 9; 15(8). pii: E1698.

3. van der Lee JH, Mokkink LB, Grootenhuis MA, Heymans HS, et al. Definitions and measurement of chronic health conditions in childhood: a systematic review. JAMA. 2007; 297:2741-51.

4. FerroMA, BoyleMH. The impact of chronic physicalillness, maternal depressive symptoms, family functioning, and self-esteem on symptoms of anxiety and depression in children. J Abnorm Child Psychol. 2015; 43(1):177-87.

5. Brehaut JC, Kohen DE, Garner RE, Miller AR, et al. Health among caregivers of children with health problems: findings from a Canadian population-based study. Am J Public Health. 2009; 99(7):1254-62.

6. Moreira JM, Bouissou Morais Soares CM, Teixeira AL, Simões e Silva AC, et al. Anxiety, depression, resilience and quality of life in children and adolescents with pre-dialysis chronic kidney disease. Pediatr Nephrol. 2015; 30(12):215362 .

7. Hill ID, Dirks MH, Liptak GS, Colletti RB, et al. Guideline for the diagnosis and treatment of celiac disease in children: Recommendations of the North American Society for Pediatric Gastroenterology, Hepatology and Nutrition, J Pediatr Gastroenterol Nutr. 2005; 40(1):1-19.

8. Esenyel S, Unal F, Vural P. Depression and anxiety in child and adolescents with follow-up celiac disease and in their families. Turk J Gastroenterol. 2014; 25(4):381-5.

9. Bai Y, Li ZS, Zou DW, Wu RP, et al. Alarm features and age for predicting upper gastrointestinal malignancy in Chinese patients with dyspepsia with high background prevalence of Helicobacter pylori infection and upper gastrointestinal malignancy: an endoscopic database review of 102665 patients from 1996 to 2006. Gut. 2010; 59(6):722-8.

10. Goodwin RD, Cowles RA, Galea S, Jacobi F. Gastritis and mental disorders. J Psychiatr Res. 2013; 47(1):128-32.

11. Rugge M, Genta RM. Staging and grading of chronic gastritis. Hum Pathol. 2005; 36(3):228-33.

12. Goodman R. The strengths and difficulties questionnaire: A research note. J Child Psychol Psychiatry. 1997; 38(5): 5816.

13. Güvenir T, ÖzbekA, Baykara B, ArkarH, etal. Psychometric properties of the turkish version of the strengths and difficulties questionnaire (SDQ). Turk J Child Adolesc Ment Health. 2008; 15(2):65-74.

14. Epstein NB, Baldwin LM, Bishop DS. The McMaster family assessment device. J Marital Fam Ther. 1983; 9(2):171-80.

15. Bulut I. Family assessment device (FAD) Manual. Ankara: Ozgüzelis Press; 1990.

16. BeckAT, Ward C, Mendelson M, MockJ, et al. An inventory for measuring depression. Arch Gen Psychiatry. 1961; 4:56171.

17. Hisli-Sahin N. Beck Depresyon Envanteri'nin geçerligi üzerine bir çalisma. Türk Psikoloji Dergisi. 1988; 6(22):11826.

18. Birmaher B, KhetarpalS, BrentD, Cully M, et al. The Screen for Child Anxiety Related Emotional Disorders (SCARED): scale construction and psychometric characteristics. J Am Acad Child Adolesc Psychiatry. 1997; 36(4):545-53.

19. Çakmakçı FK. The reliability and validity study of the screen for child anxiety- related emotional disorders (SCARED). [Unpublished Expertise Thesis]. İzmit: Faculty of Medicine, Kocaeli University; 2004.

20. Painsipp E, Wultsch T, Shahbazian A, Edelsbrunner M, et al. Experimental gastritis in mice enhances anxiety in a gender-related manner. Neuroscience. 2007; 150(3):522-36.

21. Luo J, Wang T, Liang S, Hu X, et al. Experimental gastritis leads to anxiety- and depression-like behaviors in female but not male rats. Behav Brain Funct. 2013; 9:46.

22. Mayer EA, Tillisch K, BradesiS. Review article: modulation of the brain-gut axis as a therapeutic approach in gastrointestinal disease. Aliment Pharmacol Ther. 2006; 24(6):919-33. 
e114 / Arch Argent Pediatr 2019;117(2):e110-e114 / Original article

23. Bercik P, Verdu EF, Foster JA, Macri J, et al. Chronic gastrointestinal inflammation induces anxiety-like behavior and alters central nervous system biochemistry in mice. Gastroenterology. 2010; 139(6):2102-12.e1.

24. Konturek PC, Brzozowski T, Konturek SJ. Stress and the gut: pathophysiology, clinical consequences, diagnostic approach and treatment options. J Physiol Pharmacol. 2011; 62(6):591-9.

25. Taché $Y$, Bonaz B. Corticotropin-releasing factor receptors and stress-related alterations of gut motor function. J Clin Invest. 2007; 117(1):33-40.

26. Konturek SJ. Gastric secretion from Pavlov's nervism to Popielski's histamine as direct secretagogue of oxyntic glands. J Physiol Pharmacol. 2003; 54(Suppl 3):43-68.
27. Çağan Appak Y, Yalın SapmazŞ, Doğan G, Herdem A, et al. Clinical findings, child and mother psychosocial status in functional constipation. Turk J Gastroenterol. 2017;28(6):46570

28. Sipal RF, Schuengel C, Voorman JM, Van Eck M, et al. Course of behaviour problems of children with cerebral palsy: the role of parental stress and support. Child Care Health Dev. 2010; 36(1):74-84.

29. Feldman JM, Ortega AN, Koinis-Mitchell D, Kuo AA, et al. Child and family psychiatric and psychological factors associated with child physical health problems: results from the Boricua youth study. J Nerv Ment Dis. 2010; 198(4):272-9. 